\title{
Patient engagement in a trial testing a new strategy of care for acute heart failure
}

\author{
Douglas S. Lee MD PhD, Robert Armstrong, Shanas Mohamed RN BScN; for the investigators of the COACH trial
}

Cite as: CMAJ 2018;190(Suppl 1):S34-S36. doi: 10.1503/cmaj.180462

A bout 1 million emergency department visits for heart failure occur annually in North America. ${ }^{1}$ After initial stabilization and symptom control, a decision must be made to admit or discharge a patient. About two-thirds of patients are admitted to hospital, typically for six to eight days, even though some may be amenable to early discharge. ${ }^{2}$ Of the one-third of patients who are discharged home, many are left to navigate their own follow-up care. Physicians who care for patients with acute heart failure in the emergency department often make decisions to admit or discharge using clinical judgment alone, because they do not have tools to help them to make evidence-based decisions. Often, physicians are concerned that patients will experience adverse events and be unable to access timely care if they are discharged early. This means that stable patients who could potentially be sent home safely remain in hospital. In some cases, high-risk patients may be discharged too early and may experience an adverse outcome, such as death.

We designed a study to assess whether a new approach to decision-making for patients with heart failure in the emergency department setting could assist physicians to make better discharge decisions, and whether rapid follow-up care for discharged patients could lead to better clinical outcomes. Simultaneously, our study aimed to enhance access to rapid care when patients are discharged home early. With an improved evidence base, patients can engage in more informed, shared decisions with their providers about how and where they would like their care to occur.

The Comparison of Outcomes and Access to Care for Heart Failure $(\mathrm{COACH})$ trial is a stepped-wedge randomized trial (ClinicalTrials.gov NCT02674438) comparing usual care to use of a clinical algorithm to help physicians stratify patients as high, intermediate or low risk before making a treatment and discharge decision. In the intervention arm, patients are stratified using the Emergency Heart Failure Mortality Risk Grade (EHMRG) score for their simultaneous risk of 7- and 30-day mortality in the emergency department. ${ }^{3,4}$ Early discharge home or the standard care pathway of hospital admission are recommended based on the EHMRG score. Patients who are

\section{KEY POINTS}

- Patients with acute heart failure are commonly seen in the emergency department, yet decisions about whether to admit or discharge early are often not evidence-based, and navigation of follow-up care can be difficult for patients.

- In a multicentre randomized trial, we are investigating whether decisions about early discharge can be guided effectively by patient risk-stratification, and whether a rapid postdischarge ambulatory care clinic for discharged patients can lead to improved outcomes and quality of care.

- We formed an advisory panel of patients with heart failure who assisted in study design, chose a patient-centred outcome, engaged in advising researchers during the trial and will have the opportunity to participate in fora when results are disseminated.

- Challenges were encountered in identifying the forum most conducive to meaningful dialogue for patient engagement and in obtaining consent to patient involvement from institutional review boards.

discharged early are eligible for follow-up in the Rapid Ambulatory Program for Investigation and Diagnosis of Heart Failure (RAPID-HF) transitional care clinic. ${ }^{5}$ The RAPID-HF clinic provides systematized nurse-run and cardiologist-supervised care for patients with heart failure, and facilitates any necessary testing, medications and other therapeutic measures. We aim to enrol 8000 patients in the trial.

In the intervention arm of the $\mathrm{COACH}$ trial, patients deemed to be at low risk of adverse outcomes must agree to be discharged home early during the hospital stay or directly from the emergency department. Patients and their families are central to such a decision. Therefore, our research team worked with a patient advisory panel, which we assembled before the trial began. We engaged patients at each step of the research process to provide input and guidance and to assist in the design of the trial. We used the Strategy for Patient-Oriented Research (SPOR) Patient Engagement Framework (using the guiding principles of inclusiveness, support, mutual respect and co-building) in conjunction with the Knowledge Translation 


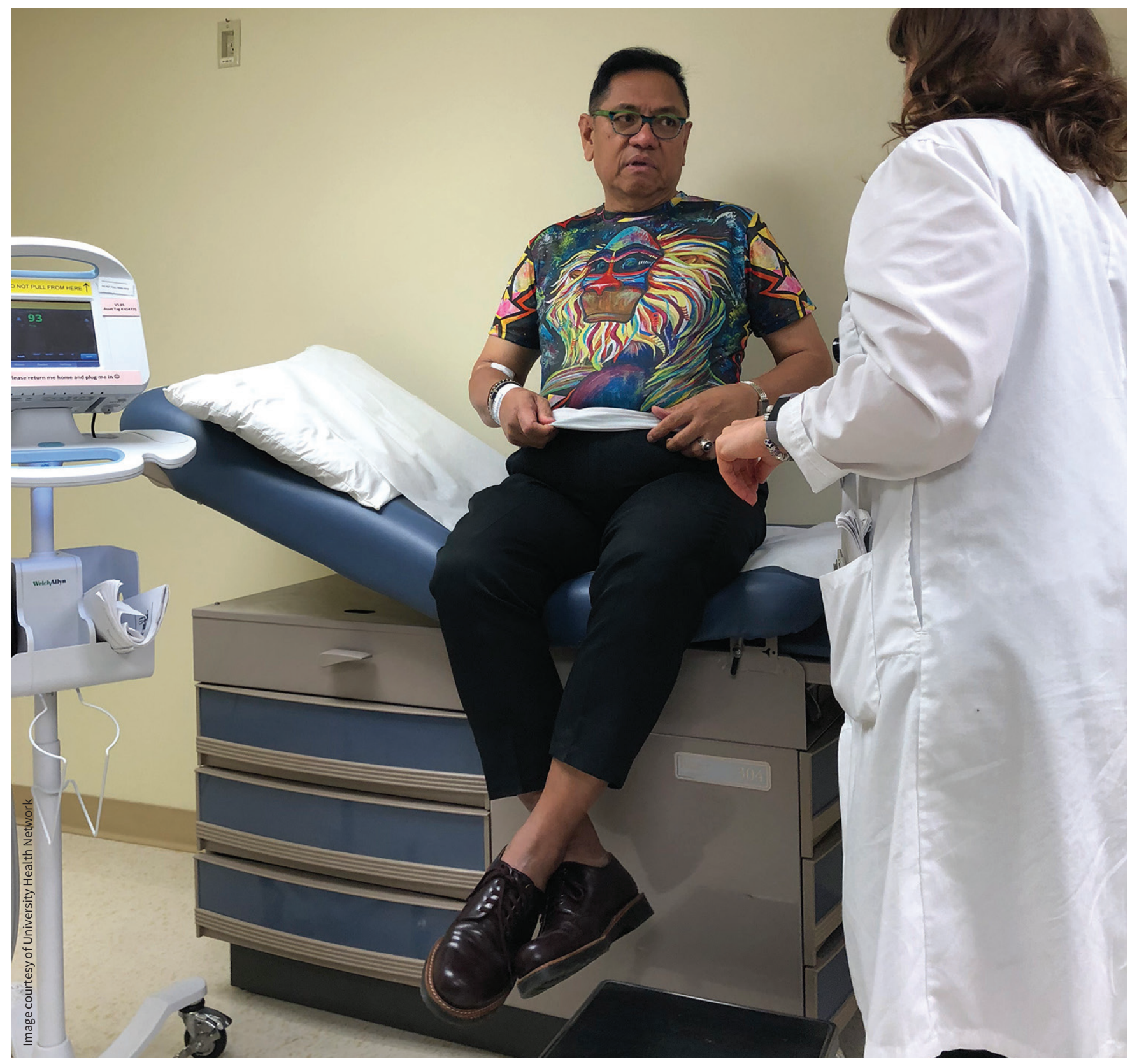

Program at St. Michael's Hospital to engage patients. ${ }^{6}$ We sought patients from broad geographic and sociodemographic backgrounds, including northern and southern Ontario, and equivalent representation of women and men from different walks of life, to form the patient advisory panel.

The panel reviewed and advised on the design of the RAPID-HF clinic intervention. Since the clinic requires patients to be active participants in their care - by being responsible for attending clinic and diagnostic test visits provided in a specialized care setting - we paid careful attention to patients' lived experiences of the emergency care they had received. Patients offered their views about shared decisionmaking related to hospital admission and how they thought the hand-off of care from the RAPID-HF clinic to their longterm provider of cardiac care should occur. Our patient advi- sory panel chose "rate of emergency department visits" as a patient-centred outcome, because frequent emergency visits disrupt patients' and families' lives and affect quality of life. While the trial is ongoing, we continue to engage our patient advisory panel for educational opportunities via formal group meetings conducted by OSSU (the Ontario SPOR SUPPORT [Support for People and Patient-Oriented Research and Trials] Unit) and via teleconference to update them on the trial's progress. The panel will have the opportunity to participate in fora when results are disseminated.

Engaging patients in all stages of research can help optimize research. Obtaining feedback from patients who actually participated in the $\mathrm{COACH}$ trial may also be important. ${ }^{7,8}$ Thus, we conducted focus-group discussions in parallel to our patient advisory meetings to gain patients' perspectives on the trial, 
reflecting on their direct experiences of being a participant in the $\mathrm{COACH}$ trial and a recipient of care in the RAPID-HF clinic. One of the patients said, "I hope that [the RAPID-HF clinic] does get carried through to other hospitals - it's really a wonderful thing to have."

At each stage of our trial, engagement with patients has enriched our understanding of how patients view their health care from the point of view of being a partner in care. However, we encountered some challenges. Research ethics boards vary in their knowledge of patient-oriented research. Some institutions forbid patient engagement until research ethics approval has been obtained, which eliminates the possibility that potential patients can participate in trial design. Some patients expressed a sense of being overwhelmed in the midst of a large group of researchers, clinicians and administrators in joint meetings. We found that smaller meetings, in which the number of patients exceeded the number of research staff, were more conducive to discussions. Despite the challenges, we were encouraged by one of our patient advisory committee members, who said, "It took a while for me to understand the exact purpose of the project and what we wanted to accomplish. As time went on I became much more comfortable as I began to understand the complexity of what you were trying to do."

Partnering with patients provided a new and important dimension to our trial of implementation of a health services intervention. The patient-engagement framework employed in the $\mathrm{COACH}$ trial could inform future similar studies to give patients a greater voice in the design of implementation trials. Furthermore, we hope our trial may give patients greater confidence that their shared decisions will be evidence-based, and high-quality care can be accessed while improving the efficiency of the care of patients with heart failure.

\section{References}

1. Storrow AB, Jenkins CA, Self WH, et al. The burden of acute heart failure on US emergency departments. JACC Heart Fail 2014;2:269-77.

2. Lee DS, Schull MJ, Alter DA, et al. Early deaths in patients with heart failure discharged from the emergency department: a population-based analysis. Circ Heart Fail 2010;3:228-35

3. Lee DS, Stitt A, Austin PC, et al. Prediction of heart failure mortality in emergent care: a cohort study. Ann Intern Med 2012;156:767-75.

4. Greig D, Austin PC, Zhou L, et al. Ischemic electrocardiographic abnormalities and prognosis in decompensated heart failure. Circ Heart Fail 2014;7:986-93.

5. Dunbar-Yaffe R, Stitt A, Lee JJ, et al. Assessing risk and preventing 30-day readmissions in decompensated heart failure: Opportunity to intervene? Curr Heart Fail Rep 2015;12:309-17.

6. Strategy for patient-oriented research - patient engagement framework. Ottawa: Canadian Institutes of Health Research; 2014. Available: www.cihr -irsc.gc.ca/e/48413.html (accessed 2018 Aug. 30).

7. Domecq JP, Prutsky G, Elraiyah T, et al. Patient engagement in research: a systematic review. BMC Health Serv Res 2014;14:89.

8. Esmail L, Moore E, Rein A. Evaluating patient and stakeholder engagement in research: moving from theory to practice. J Comp Eff Res 2015;4:133-45.
More information on this project is available at www.ossu.ca/IMPACTAwards.

\section{Competing interests: None declared.}

This article was solicited and has been peer reviewed.

Affiliations: The Peter Munk Cardiac Centre (Lee, Mohamed) and the Joint Department of Medical Imaging (Lee), University Health Network; Institute for Clinical Evaluative Sciences (Lee); Institute of Health Policy, Management and Evaluation, University of Toronto (Lee), Toronto, Ont.; patient partner (Armstrong), Caledon, Ont.
Contributors: Douglas Lee drafted the manuscript, which Robert Armstrong and Shanas Mohamed revised. All authors gave final approval of the version to be published and agreed to be accountable for all aspects of the work.

Funding: The study was supported by a Strategy for Patient-Oriented Research (SPOR) grant from OSSU (the Ontario SPOR SUPPORT [Support for People and Patient-Oriented Research and Trials] Unit) and a Foundation Grant from the Canadian Institutes of Health Research (no. FDN 148446). Douglas Lee is supported by a Mid-Career Investigator Award from the Heart and Stroke Foundation and the Ted Rogers Chair in Heart Function Outcomes, a joint hospital-university chair of the University Health Network and the University of Toronto.

Disclaimer: The Institute for Clinical Evaluative Sciences (ICES) is supported in part by a grant from the Ontario Ministry of Health and Long-Term Care. The opinions, results and conclusions are those of the authors and no endorsement by the Ministry of Health and Long-Term Care or by ICES is intended or should be inferred.

Correspondence to: Douglas Lee, dlee@ices.on.ca 